\title{
Substratos alternativos para propagação vegetativa de Psychotria ipecacuanha
}

\section{(Brotero) Stokes}

\author{
Alternative substrates for vegetative propagation of Psychotria ipecacuanha (Brotero) Stokes \\ Sustratos alternativos para la propagación vegetativa de Psychotria ipecacuanha (Brotero) Stokes
}

Recebido: 17/04/2021 | Revisado: 25/04/2021 | Aceito: 30/04/2021 | Publicado: 14/05/2021

\author{
Osmar Alves Lameira \\ ORCID: https://orcid.org/0000-0001-8370-8562 \\ Embrapa Amazônia Oriental, Brasil \\ E-mail: osmar.lameira@embrapa.br \\ Meiciane Ferreira Campelo \\ ORCID: https://orcid.org/0000-0001-7511-4377 \\ Universidade Federal do Pará, Brasil \\ E-mail: meicianecampelo@gmail.com \\ Ruanny Karen Vidal Pantoja Portal Moreira \\ ORCID: https://orcid.org/0000-0002-9566-3741 \\ Universidade Federal do Pará, Brasil \\ E-mail: ruanny_vidal@ hotmail.com \\ Simone de Miranda Rodrigues \\ ORCID: https://orcid.org/0000-0001-5717-785X \\ Embrapa Amazônia Oriental, Brasil \\ simone.rodrigues@embrapa.br \\ Allan Cristiam Santos Ramires \\ ORCID: https://orcid.org/0000-0002-0953-7221 \\ Universidade Federal Rural da Amazônia, Brasil \\ E-mail: allanramires15@gmail.com
}

\begin{abstract}
Resumo
O objetivo deste estudo foi determinar entre diferentes substratos qual proporciona melhores condições para propagação vegetativa de ipeca via estaca de raiz, otimizando assim este processo para que se atenuem as limitações da produção em escala comercial da espécie. Foi estabelecido experimento em casa de vegetação, os segmentos de raiz de ipeca foram introduzidos em bandejas, preenchidas com diferentes substratos: vermiculita, areia, fibra de coco, serragem, palha de arroz e composto vegetal. O delineamento experimental utilizado foi inteiramente casualizado com seis tratamentos e oito repetições, sendo cada repetição composta por um segmento de raiz, totalizando 48 unidades experimentais. Os resultados demonstraram que a escolha do substrato exerceu influência na ocorrência de lançamento de parte aérea. Os substratos que apresentam maiores percentuais de presença de raiz foram respectivamente serragem, palha de arroz, vermiculita e composto vegetal, seguido dos substratos fibra de coco e areia. As variáveis número de folhas e comprimento médio da parte aérea revelaram respostas estatisticamente semelhantes entre os tratamentos e para as variáveis comprimento de raiz e número de raiz não foi verificado diferenças significativas. A propagação vegetativa via estaca de raiz é promissora quando se utiliza o substrato serragem que proporciona crescimento satisfatório, considerando as respostas mutuas das características morfológicas analisadas em mudas de Psychotria ipecacuanha (Brotero) Stokes em apenas 10 semanas.
\end{abstract}

Palavras-chave: Plantas medicinais; Ipeca; Reprodução vegetativa.

\begin{abstract}
The objective of this study was to determine between different substrates which provides better conditions for vegetative propagation of ipecac via root cut, thus optimizing this process so that the limitations of the commercial scale production of the species are attenuated. An experiment was established in a greenhouse, the segments of ipecac root were introduced in trays, filled with different substrates: vermiculite, sand, coconut fiber, sawdust, rice straw and vegetable compost. The experimental design used was completely randomized with six treatments and eight repetitions, each repetition being composed of a root segment, totaling 48 experimental units. The results showed that the choice of substrate had an influence on the occurrence of shoot release. The substrates with the highest percentage of root presence were sawdust, rice straw, vermiculite and vegetable compost, respectively, followed by the substrates coconut fiber and sand. The variables number of leaves and average length of the aerial part revealed statistically similar responses between treatments, and for the variables length of root and number of root there were no significant differences. The vegetative propagation via root cut is promising when using the sawdust substrate that provides satisfactory growth, considering the mutual responses of the morphological characteristics analyzed in Psychotria ipecacuanha (Brotero) Stokes seedlings in just 10 weeks.
\end{abstract}


Keywords: Medicinal plants; Ipeca; Vegetative reproduction.

\section{Resumen}

El objetivo de este estudio fue determinar entre diferentes sustratos cuál brinda mejores condiciones para la propagación vegetativa de la ipecacuana mediante corte de raíz, optimizando así este proceso de manera que se atenúen las limitaciones de la producción a escala comercial de la especie. Se estableció un experimento en invernadero, se introdujeron los segmentos de raíz de ipecacuana en bandejas, llenas de diferentes sustratos: vermiculita, arena, fibra de coco, aserrín, paja de arroz y abono vegetal. El diseño experimental utilizado fue completamente al azar con seis tratamientos y ocho repeticiones, estando cada repetición compuesta por un segmento de raíz, totalizando 48 unidades experimentales. Los resultados mostraron que la elección del sustrato influyó en la aparición de la liberación de brotes. Los sustratos con mayor porcentaje de presencia de raíces fueron aserrín, paja de arroz, vermiculita y compost vegetal, respectivamente, seguidos de los sustratos fibra de coco y arena. Las variables número de hojas y longitud promedio de la parte aérea revelaron respuestas estadísticamente similares entre tratamientos, y para las variables longitud de raíz y número de raíz no hubo diferencias significativas. La propagación vegetativa por corte de raíz es prometedora cuando se utiliza el sustrato de aserrín que proporciona un crecimiento satisfactorio, considerando las respuestas mutuas de las características morfológicas analizadas en plántulas de Psychotria ipecacuanha (Brotero) Stokes en tan solo 10 semanas.

Palabras clave: Plantas medicinales; Ipeca; Producción vegetativa.

\section{Introdução}

A Psychotria ipecacuanha (Brotero) Stokes é conhecida popularmente como ipeca, ipecacuanha e poaia (Neves et al., 2017). Pertencente à família Rubiácea, pode ser encontrada em florestas e regiões de vegetação adensada em boa parte do território nacional, com maior ocorrência no estado do Mato Grosso especificamente no município de Cáceres. Sua importância esta voltada para a atividade farmacológica dos alcalóides, emetina e cefalina encontrados na planta, que atuam como emético, amebicida, expectorante e antiflamatório (Neves et al., 2019).

Devido ao seu alto potencial econômico a ipeca sofreu um grande processo extrativista no século XVI juntamente com a abertura de fronteiras agrícolas e desmatamento florestal que culmina de forma negativa para a perpetuação e proliferação desta espécie devido ao processo de erosão genética e também falta de habitat natural (Neves, 2019), além do mais a propagação da P. ipecacuanha por semente torna-se inviável devido sua fisiologia, cujo processo germinativo varia de 3 a 6 meses para ocorrer e apresentar baixo índice de germinação, somando a desvantagem para o plantio comercial por promover variabilidade genética gerando um cultivo desuniforme, o que dificulta ainda mais o cultivo da espécie por esse método. Portanto, faz-se necessário o uso de outros métodos de propagação (Lameira, 2002).

Sendo assim, a propagação assexuada da espécie apresenta-se como uma alternativa, pois consiste na reprodução de indivíduos através de partes vegetativas da planta com o benefício de se perpetuar características genéticas da planta doadora e em tempo inferior a propagação via semente. Diversas partes da planta podem ser utilizadas como caule, rebentos (brotos) ou raiz. A eficácia do método só e possível pelo fato dos tecidos vegetais possuírem células com duas funções fundamentais. Segundo Vignolo (2014), a totipotência consiste na recriação de um indivíduo, mantendo a sua informação genética, enquanto a desdiferenciação celular é a capacidade que as células possuem de se especializarem e retornarem a condições meristemáticas para gerar novos pontos de crescimento.

O sucesso da propagação vegetativa via estaca de raiz pode ser diretamente influenciada por fatores abióticos, sendo o substrato um fator determinante para o desenvolvimento da muda. Um substrato adequado deve proporcionar a planta: sustentação, boa porosidade e umidade adequada, influenciando diretamente na taxa de pegamento e desenvolvimento das mudas após o plantio no campo (Vence 2008, Silva et al., 2017).

Os substratos comerciais apresentam qualidades físico-química satisfatórios para a produção de mudas (Monteiro Neto et al., 2018), porém, pode ser inviável por onerar os custos de produção. Portanto, é interessante ter como alternativa a utilização de substratos confeccionados a partir de materiais disponíveis e acessíveis a cada região (Silva et al., 2019).

Apesar da importância da espécie e do potencial econômico mencionado, estudos sobre o cultivo da ipecacuanha para produção em escala comercial ou até mesmo para reinserção no seu habitat natural ainda são escassos. 
Contudo o estudo tem como objetivo determinar dentre diferentes substratos qual proporciona melhores condições para propagação vegetativa de ipeca via estaca de raiz, otimizando assim este processo para que se atenuem as limitações da produção em escala comercial da espécie.

\section{Metodologia}

\subsection{Coleta do material}

O experimento emprega como fonte de material botânico a Psychotria ipecacuanha (Brot.) Stokes., registrado no herbário IAN (4093). Espécie estabelecida in vivo e ex vitro no Banco Ativo de Germoplasma de Ipeca pertencente ao Horto de Plantas Medicinais e Aromáticas da Embrapa Amazônia Oriental, localizada no município de Belém, Estado do Pará-BR, com temperaturas médias, máximas e mínimas de $26.4{ }^{\circ} \mathrm{C}, 31.8{ }^{\circ} \mathrm{C}, 22.9{ }^{\circ} \mathrm{C}$, respectivamente, umidade relativa de $84 \%$ e índice chuvoso de $3.001 \mathrm{~mm}$ (Bastos et al., 2002).

\subsection{Montagem do experimento}

Inicialmente realizou-se a coleta de raízes, lavagem em água corrente para retirada de resíduos de solo, em seguida foram seccionadas transversalmente para obtenção de explantes (segmento de raiz) de aproximadamente $3 \mathrm{~cm}$ de comprimento e diâmetro de $0,15 \mathrm{~cm}$. O experimento foi montando em condições de casa de vegetação onde foram inoculados em bandejas de plásticos contendo células preenchidas com os seguintes substratos: vermiculita, areia, fibra de coco, serragem, palha de arroz e composto vegetal, todos previamente umedecidos com água, e durante a condução do experimento, sempre que necessário, foi realizado irrigações com auxilio de regador.

Os experimentos em estudo foram conduzidos por meio de método quantitativo e qualitativo da metodologia cientifica (Pereira et al., 2018). O delineamento experimental foi inteiramente casualizado com seis tratamentos e oito repetições, sendo cada repetição composta por um segmento de raiz, totalizando 48 unidades experimentais. $\mathrm{O}$ experimento foi monitorado por 10 semanas, percentuais de lançamento da parte aérea foram obtidos semanalmente, os registros das variáveis números de folhas, comprimento da parte aérea, presença e ausência de raiz, número de raízes e comprimento da maior raiz foi extraído na10 semana.

Os dados coletados foram submetidos a analise de variância e posterior teste de médias Scott-Knott ao nível de 5\% de probabilidade, utilizando o programa estatístico SISVAR (Ferreira, 2011) e elaboração de gráficos expondo dados com valores em percentuais com o auxilio do Software Excel.

\section{Resultados e Discussão}

Os resultados encontrados demonstram que a escolha do substrato exerceu influência na ocorrência de lançamento da parte aérea, em segmentos de raiz de ipeca ao longo de 10 semanas, nota-se que na primeira e segunda semana não houve nenhum lançamento da parte aérea. A partir da terceira semana exceto pelo substrato fibra de coco que teve o lançamento da parte aérea tardio começando apenas na sétima semana (50\%) os demais substratos já iniciavam o lançamento da parte aérea, cujo desenvolvimento ocorreu de maneira distinta de acordo com os substratos no período estudado como mostra a Figura 1. Segundo Lameira (2002), as condições ambientais apresentadas na Amazônia Oriental possibilitam que o enraizamento e formação da parte aérea dessa espécie ocorram com no mínimo 20 dias após o plantio. 
Figura 1. Porcentagem $(\%)$ de lançamento da parte aérea na propagação vegetativa de ipeca via estaca de raiz em diferentes substratos cultivadas em casa de vegetação.

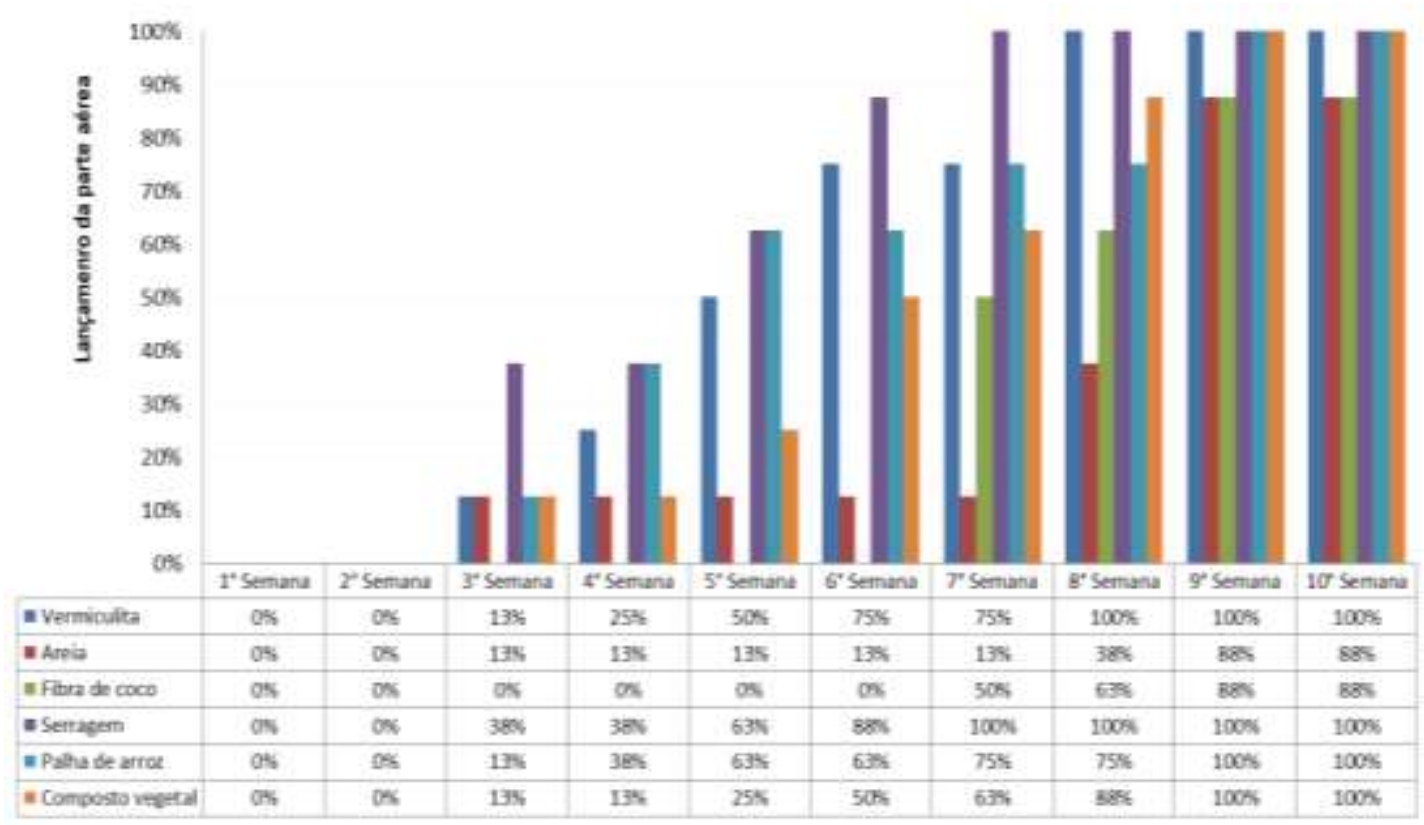

Fonte: Autores (2020).

Ressalta-se que o substrato serragem, a partir da terceira semana já apresenta lançamento da parte aérea em $38 \%$ das estacas, maior percentual obtido entre os substratos neste período, ao decorrer das semanas, tal percentual exibia continuo aumento $38 \%$ ( $4^{\circ}$ semana), $63 \%$ ( $5^{\circ}$ semana), $88 \%$ ( $6^{\circ}$ semana), sendo o primeiro substrato no qual as estacas atingiram $100 \%$ de surgimento da parte aérea na sétima semana, seguido do substrato vermiculita com 100\% na oitava e palha de arroz e composto vegetal com $100 \%$ na nona semana, logo a serragem destaca-se de forma superior aos demais substratos para esta variável (Figura1). A presença da serragem aumenta características como aeração, estrutura do substrato e retenção de água, o que permitiu o melhor desenvolvimento das mudas (Dionísio et al., 2019).

Na décima semana, ao final da avaliação dentre os seis substratos, apenas serragem, vermiculita, palha de arroz e composto vegetal alcançaram $100 \%$ de surgimento da parte aérea de ipeca. Os substratos areia e fibra de coco apresentaram ambas $88 \%$ de surgimento desta variável mostrando-se inferiores aos demais. A fibra de coco em sua composição possui substâncias como tanino, cloreto de potássio e cloreto de sódio, que quando presentes em altas concentrações podem ser prejudiciais ao desenvolvimento das plantas, principalmente nas fases iniciais (Carrijo, 2002).

Resultados compatíveis aos da variável lançamento da parte aérea foram obtidos na porcentagem de enraizamento, como se observa na Figura 2, onde os substratos que apresentam maiores percentuais de presença de raiz foram, respectivamente, serragem (88\%), palha de arroz (88\%), vermiculita (75\%) e composto vegetal (50\%), seguido dos substratos fibra de coco e areia com os menores percentuais de enraizamento (13\%). Tal fator pode estar relacionado à areia possuir uma densidade grande e reduzida porosidade, gerando deficiência na aeração das mesmas (Schmitzet al., 2002). 
Substratos adequados devem promover desenvolvimento do sistema radicular e devem conter características físicoquímicas para permitir aeração, retenção de água, nutrientes e oxigênio para mudas (Silva et al., 2017). Para Monteiro et al. (2017) uma das principais características físicas a serem consideradas na caracterização dos substratos é a capacidade de retenção de água.

Embora não tenham ocorrido diferenças estatísticas das variáveis em meio aos substratos em análise, para o comprimento da parte aérea, o composto vegetal apresentou à maior media geral $(3,08 \mathrm{~cm})$, seguido dos substratos vermiculita $(2,85 \mathrm{~cm})$, serragem $(2,77 \mathrm{~cm})$, fibra de coco $(2,46 \mathrm{~cm})$, areia $(1,93 \mathrm{~cm})$ e palha de arroz $(1,90 \mathrm{~cm})$. Andrade et al. $(2021) \mathrm{em}$ estudo com rubiaceae nota que o crescimento das mudas foram interferidas pelos fatores recipiente e substrato o que pode resultar em qualidades distintas das mudas.

Quanto ao número de folhas, à ordem decrescente das medias obtidas foi 3,33;3,12;2,87; 2,62; 2,42 e 2,00, respectivamente aos substratos serragem, vermiculita, composto vegetal, fibra de coco, palha de arroz e areia (Figura 3A). De acordo com Klein (2015), o uso de areia na formulação dos substratos pode ser de grande interesse para a produção de mudas, por ser um material de constante disponibilidade e de baixo custo, entretanto, seu uso na propagação vegetativa de ipeca não foi tão satisfatório para o desenvolvimento das plântulas.

Para a variável, comprimento de raiz os maiores valores médios consequentemente, foram obtidos para os substratos palha de arroz, serragem, vermiculita e composto vegetal referente às médias 1,59;1,16;1,0 e $0,55 \mathrm{~cm}$, respectivamente. E para a variável, número de raiz o substrato vermiculita seguido da palha de arroz, serragem e composto vegetal apresentaram médias de 2,0; 1,86; 1,71 e 1,0 respectivamente (Figura 3B).

Figura 3. A-B: Valores médios do comprimento da parte aérea, número de folhas, comprimento da maior raiz e número de raízes de mudas de ipeca após propagação vegetativa em diferentes substratos. Embrapa, Belém-PA, 2020.

A

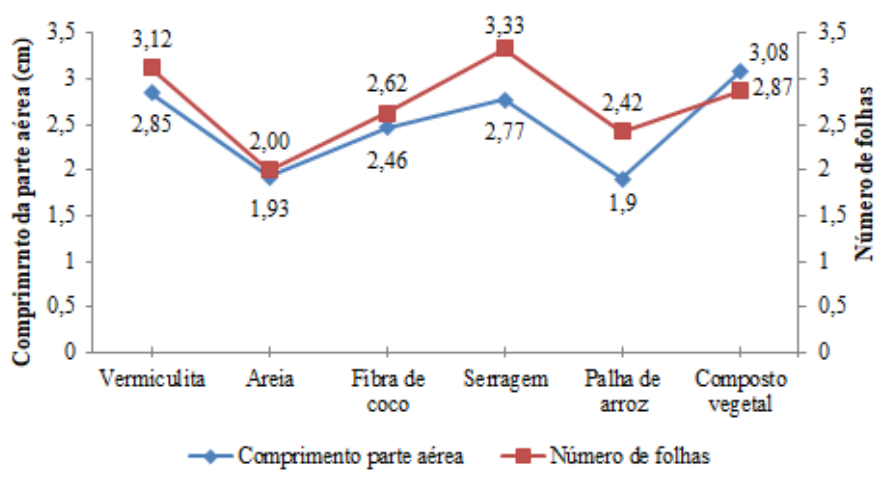

B

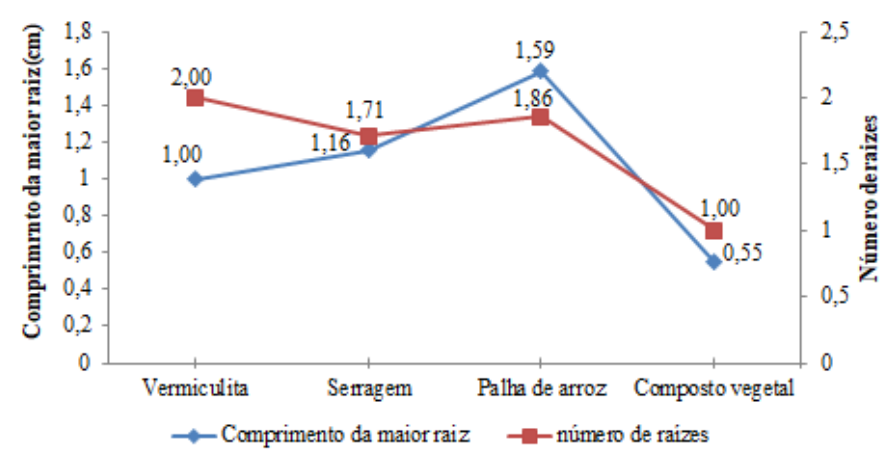

Fonte: Autores (2020). 
Embora a aplicação da ANOVA em quatro variáveis, comprimento da parte aérea, número de folhas, comprimento e número de raízes, não apresentou diferenças estatísticas, o substrato serragem se destaca nestas variáveis constantemente entre as três maiores médias obtidas, além, de alcançar a maior porcentagem de enraizamento (88\%) no período avaliado, observando que na sétima semana, diferentemente dos demais, já havia alcançado 100\% de lançamento da parte aérea, com isso, este substrato se destaca com o desenvolvimento mais expressivo comparado aos demais substratos em estudo.

\section{Conclusão}

A propagação vegetativa via estaca de raiz é promissora quando se utiliza o substrato serragem que proporciona crescimento satisfatório considerando respostas mútuas das características morfológicas analisadas em mudas de Psychotria ipecacuanha (Brotero) Stokes em apenas 10 semanas, período relevantemente inferior à propagação assexuada.

\section{Referências}

Andrade, C. R.; Melo, B. M. R. De.; Mendes, C. T. E.; Castro, D. G.; Coelho, E. L.; \& Francisco, K. C. P. (2021). Crescimento e qualidade de mudas de diferentes cultivares de cafeeiro sob diferentes substratos e recipientes. Research, Societyand Development, v. 10, n. 2, e2810212073, 2021. DOI: http://dx.doi.org/10.33448/rsd-v10i2.12073.

Bastos, T. X.; Pacheco, N. A.; Nechet, D.; \& Sá, T. D. A. (2002). Aspectos climáticos de Belém nos últimos cem anos, Belém: Embrapa Amazônia Oriental. Documentos, 128, 31p. ISSN 1517-2201. CDD: 551.6098115.

Carrijo, O. A.; Liz, R. S.; \& Makishima, N. (2002). Fibra da casca do coco verde como substrato agrícola. Horticultura brasileira, 20(4), 533-535. Doi:http://dx.doi.org/10.1590/S0102-05362002000400003.

Dionisio, L. F. S.; Auca, E. C.; Bardales-Lozano, R. M.; Schwartz, G.; Rodrigues, R. P.; Corvera-Gomringer, R. (2019). ProdutionofBertholletiaexcelsaHumb. \& Bonpl., (Lecythidaceae) seedlings in microenvironmentsunderdiferentsubstrates.Revista Brasileira de Ciências Agrárias, Recife, 14(3),e5847.DOI: 10.5039/agrária.v14i3a5847.

Ferreira, D.F. (2011) Sisvar: A computer statistical analysis system. Ciência e Agrotecnologia, Lavras, 35(6), 1039-1042. Doi: https://doi.org/10.1590/S141370542011000600001 .

Klein, C. (2015). Utilização de substratos alternativos para produção de mudas. Revista Brasileira de Energias Renováveis, v. 4, n. 4363.Doi:https://doi.org/10.5380/rber.v4i3.40742.

Lameira, O. A. Cultivo da ipecacuanha [Psychotriaipecacuanha (Brot.) Stokes]. Embrapa Amazônia Oriental-Circular Técnica (INFOTECA-E), 2002. https://www.infoteca.cnptia.embrapa.br/bitstream/doc/405771/1/Circ.tec.28.pdf>.

Monteiro Neto, J. L. L.; Araújo, W.F.; Vilarinho, L. B. B. O.; Nunes,T. K.; Silva, E. S.; Maia, S. S.; Albuquerque, J. A. A.; Chagas, E. A.; Siqueira, R. H. S.; Abbannto-Rodrigues, C. (2018). Seedlinngsproductionoftwotomato (SolanumlicopersicumL.) cultivarsunderdiferentenvironnmenntsandsubstrates. Revista Acta Agronómica, 67(2), 270-276.Doi:http://dx.doi.org/10.15446/acag.v67n2.67943.

Monteiro, A. B.; Pereira, I. Dos S.; Stocker, C. M.; Peres, M. A.; Martinazzo, R.; Bamberg, A. L.; Timm, L. C. (2017). Substratos a base de lodo de esgoto solarizado e pirolizado para produção de mudas de alface. Revista Congrega Urcamp, Bagé, 13.

Neves, R. L. P.; Lameira, O. A.; Oliveira, M. S. P.; Souza, F. I. B.; Monfort, L. E. F.; Correiai, R. G. (2017). Caracterização e avaliação morfológica da parte aérea de acessos de Psychotriaipecacuanha (Brot.) Stokes (IPECA). Revista Cubana de Plantas Medicinales,22,1. $<$ https://www.researchgate.net/profile/Raphael_Lobato_Prado_Neves/publication/319455497_Characterization_and_morphological_evaluation_of_aerial_part s_of_accessions_of_Psychotria_ipecacuanha_Brot_Stokes_IPECA/links/5a686a19aca272de02f459c7/Characterization-and-morphological-evaluation-ofaerial-parts-of-accessions-Psychotria-ipecacuanha-Brot-Stokes-IPECA.pdf >.

Neves, R. L. P.; Lameira, O. A.;Medeiros, A. P. R.; Pires, H. C. G.; Oliveira, M. G. DE; Germano, C. M.; Leão, F. M.(2019). Caracterização e avaliação morfológica da parte aérea de acessos de Psychotriaipecacuanha (IPECA).1-388-416. Ponta Grossa-PR: EditoraAtena. <https://www.atenaeditora.com.br/post-artigo/18755>.

Pereira, A. S., Shitsuka, D. M., Parreira, F. B., \& Shitsuka, R. (2018). Metodologia da pesquisa científica [recurso eletrônico [eBook]. Santa Maria. Ed. UAB/NTE/UFSM. https://repositorio. ufsm. br/bitstream/handle/1/15824/Lic_Computacao_MetodologiaPesquisa-Cientifica. pdf.

Schmitz, J. A.; Souza, P. V.; Kämpf, A. N. (2002). Propriedades químicas e físicas de substratos de origem mineral e orgânica para o cultivo de mudas em recipientes. Ciência Rural, 32(6), 937-944. Doi: https://doi.org/10.1590/S0103-84782002000600005.

Silva, A. C. D.; Smiderle, J. O.; Oliveira, J. M. F.; Silva, T. J. (2017). Tamanho da semente e substratos na produção de mudas de açaí. Advances in Forestry Science,4, (4), 151-156. Doi: 10.34062/afs.v4i4.4590.

Silva, W. V.; Costa, A. C.; Silva, V. L. (2019). Substratos na produção de mudas de cultivares de maracujazeiro azedo. Revista Cultivando o saber, 12 (1), 11 24.

Vence, L. B. (2008). Disponibilidad de agua-aire em sustratos para plantas. Ciência Delsuelo, 26(2), 105-114. <http://suelos.org.ar/publicaciones/vol_26n2/26$2 \% 20$ Vence.pdf>. 
Research, Society and Development, v. 10, n. 5, e49210515158, 2021

(CC BY 4.0) | ISSN 2525-3409 | DOI: http://dx.doi.org/10.33448/rsd-v10i5.15158

Vignolo,G.K.;Picolotto,L.;Gonçalves,M.A.;Pereira,I.DOSS.;Antunes, L.E.C.(2014). Presença de folhas no enraizamento de estacas de amoreira-preta. Ciência Rural, 44 (3), 467-472.Doi: http://dx.doi.org/10.1590/S0103-84782014000300013. 